\title{
FAMOUS: A Network Architecture for Delivering Multimedia Services to FAst MOving USers
}

\author{
Filip De Greve, Bart Lannoo, Liesbeth Peters, Tom Van Leeuwen, \\ Frederic Van Quickenborne, Didier Colle, Filip De Turck, Ingrid \\ Moerman, Mario Pickavet, Bart Dhoedt, Piet Demeester \\ Department of Information Technology (INTEC), Ghent University \\ Sint-Pietersnieuwstraat 41, B-9000 Gent, Belgium. \\ Tel.: +32926499 70, Fax: +3292649960 \\ \{name.surname@intec.ugent.be\}
}

September 15, 2004

\begin{abstract}
When today's commuters in the train or in a car want to access the Internet, they see themselves restricted to simple web surfing or e-mail. Interactive multimedia services, like online gaming or video conferencing are still unavailable to them, even with promising new technologies like UMTS or WiMAX. The impact of high bit rate multimedia traffic on the access network and aggregation network is an important topic, that has not been addressed in enough detail before. We designed a network architecture for offering these multimedia services to fast moving users. We refer to the overall network architecture as the FAMOUS network architecture, which consists of two parts: (i) an access network part which has to deal with large number of users, asking for a high bandwidth, while experiencing a high handoff frequency and (ii) an aggregation network part which has to deal with dynamic tunnels of very high bandwidth, while experiencing a low handoff frequency. In this paper, we detail the FAMOUS architecture, together with optimized handoff strategies, an optical switching architecture, a design methodology for dimensioning aggregations networks and automatic tunnel pre-configuration and activation. Moreover, performance results of these mentioned aspects will be presented.
\end{abstract}

Keywords: broadband, wireless, fast moving users, fast handoff, dynamic traffic engineering, Ethernet

\section{Introduction}

\subsection{Why BROADBAND MOBILE COMMUNICATION?}

Nowadays, a lot of multimedia applications are taken for granted in fixed networks. These applications require a high level of Quality of Service (QoS) and generally are characterized by high bandwidth requirements and low latencies, which can currently only be offered by fixed broadband access technologies. The challenge future and current telecom operators are facing is to examine how this bandwidth and quality can also be provided in wireless cellular networks.

(C) 2005 Kluwer Academic Publishers. Printed in the Netherlands. 
The number of Internet users is still expanding rapidly (from 688 Million users end 2003 [1] to 800 Million users in September 2004 [2], while the number of fixed broadband Internet users (DSL, cable, FTTH) is increasing even more rapidly. End 2003 there were about 100 Million broadband users [3], which is $15 \%$ of all Internet users. However, when looking at the mobile subscriber statistics which reveal 1511 Million subscribers in June 2004 [4], we may conclude that the mobile terminal is without any doubt the most popular terminal. These numbers clearly indicate the great potential of broadband services for mobile terminals.

Today, organizations like IEEE and 3GPP are establishing specifications for new wireless technologies which have to meet the bandwidth requirements of tomorrow (e.g. UMTS and WiMAX). Unfortunately, the needs of the fast moving users, e.g. in the car or on the train, have never been taken into account: the faster they move, the more their available bandwidth decreases. In densely populated areas, such as cities and business centers, there is an evolution towards smaller cell sizes in order to cope with the higher demands for bandwidth. But fast moving users are still transferred to larger cells, which drastically reduces their available bandwidth. In other words, current wireless network technologies are not capable to meet the demand for high bandwidth at vehicular speeds.

In a cellular network, a high bandwidth capacity can only be achieved by reducing the cell size, hereby efficiently reusing the limited available frequency spectrum. In future broadband wireless networks, cellular networks will hence consists of very small cells (typically a few 100m), not only in densely populated areas but also along the roads and train tracks in rural areas.

\subsection{Current technology Deployments}

Currently trials and early commercial releases that deliver broadband internet to passengers in fast moving vehicles are emerging. However, there is no consensus on a widely deployed platform and different technologies are used to realize the wireless connection with global coverage and broadband capacity. Internet connections via satellites are used for in-flight Internet services on airplanes [5] but also for trains [6], wireless connections via cellular techniques such as GPRS or UMTS are explored [7], Wi-fi or WiMAX solutions are mainly explored for car scenarios [8] and wireless connection with directed on-shore antennas is used to deliver onboard internet on ferries [9]. Trials for hybrid solutions that can use $\mathrm{Wi}-\mathrm{Fi}$ when the vehicle is in the vicinity of a hotspot and GPRS throughout the rest of the trip [10] are also investigated. In Table I, three current technologies that are used in the 
Table I. Three technologies compared

\begin{tabular}{c|c|c|c} 
& Bandwidth & Latency & Handoff \\
\hline Satellite & \pm & - & + \\
Cellular & - & \pm & + \\
Wi-fi and WiMAX & + & + & -
\end{tabular}

above mentioned references, are compared to each other with respect to their capabilities to deliver broadband services to fast moving users. None of the technologies can meet all requirements: high bandwidth, low latency and fast handoff.

\section{The FAMOUS network architecture}

The network architecture proposed in this paper will be referred to as FAMOUS (= FAst MOving USers). For the design of the FAMOUS network architecture that is responsible for the data transport between the fast moving users and the service provider, we propose a partitioned network solution as depicted on Figure 1). Two specific sub-problems, namely the development of the aggregation network and the development of the access network, will be discussed in detail. This section will continue with a short account on the general concept and an overview of the specific problems that need to be addressed for the different network parts.

Because of the inherent small cells in broadband mobile networks a dense placement of wireless antennas is required along the trajectory of the vehicles. The moving vehicle will remain connected to the closest wireless base station in the vicinity while passengers are connected to the internal vehicle network. These wireless base stations are bundled in access networks and while vehicles are moving, the vehicle's wireless connections hop from one wireless antenna to another. Inside the access network a micromobility protocol will be responsible for maintaining a seamless connection between the moving users and the infrastructure of the aggregation network (as discussed in Section 4).

The mobility of users is not limited to a single access network. The connection with the fast moving user will hop from access network to access network and this will imply that the connection point between the access network and the aggregation network, named the access gateway (AGW), will change concordingly. The aggregation network is responsible for the transport of data traffic between the AGWs and the service providers and a macromobility protocol is required to manage mobility between SGWs and AGWs in the aggregation 


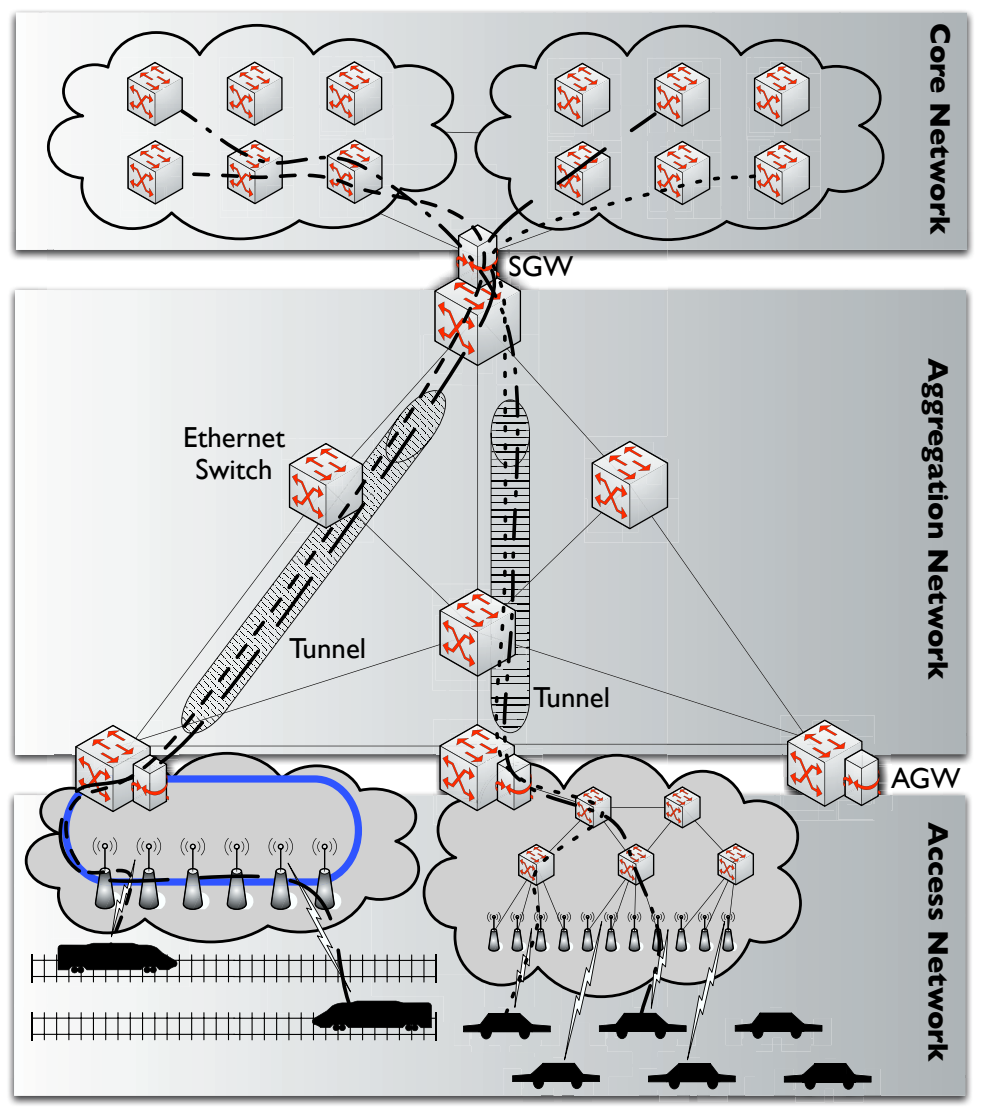

Figure 1. Network architecture and terminology

network (as discussed in Section 5). While the access networks will handle traffic of the wireless users individually, the aggregation network will only take aggregated traffic flows into account and transport them towards the service provider domains. Examples of service providers are telephone operators, Internet service providers (ISPs) and content providers (e.g. to offer Video-on-Demand services). The connection between the service providers and the aggregation network is realized by service gateways (SGWs), e.g., PSTN switches for telephone operators or Broadband Access Servers (BAS) for ISPs or content servers.

In this network the AGWs and the SGWs are Layer 3 aware devices and they split the network in three separated IP network parts. This is concordant with the current evolution in access network design towards all-IP based network architectures. In the following sections these parts of the FAMOUS network architecture are briefly introduced. 
- Access Networks

Due to the mobility of a car or a train, a handoff protocol is needed in the access network. This protocol has to ensure a continuous network connection to the mobile users, each time they visit an other base station cell.

The IETF has therefore developed the Mobile IPv6 protocol [11] which allows IP hosts to remain connected to the Internet while moving, changing its local IP address on each handoff. The delay between the moment the Mobile IP aware host has associated with the new base station, configured its connection to the network and updated its location, may be substantial, e.g. in the order of seconds. It is well known that in the case of high handoff frequency and small cell sizes, the Mobile IP protocol won't be able to follow the frequent change in connections between base stations and will ultimately fail.

Mobile IP is therefore not suited as a handoff protocol for delivering high bandwidth high quality multimedia services to fast moving users within the same access network. In Section 4 we propose dedicated solutions within one access network.

- Aggregation Networks

Nowadays, fixed broadband networks are not optimally designed to cope with the traffic of users moving at vehicular speed. Therefore, an efficient way for designing and managing an aggregation network under dynamically changing traffic conditions is proposed. We will detail the proposed network architecture and two important parts of the management system for the aggregation network: the protocols for automatic service realization and an efficient method for calculating the capacity assignment and the path determination. It has been proven in [12] that networks lacking the ability to adapt dynamically according to fast moving traffic demands, are significantly more expensive. As indicated in the previous paragraph, Mobile IP is a valid choice for environments with rather slow handoff frequencies. Therefore Mobile IP is used to follow the connections of moving vehicles in the aggregation network where the vehicle's connections will hop regularly from access network to access network.

\section{- Core Networks}

The aggregation network is connected to the core network via a Service Gateway (SGW). This SGW has similar functionality as the Broadband Remote Access Server (BRAS) in a classical access network: user IP packets are forwarded to the right provider and 
a distinction can be made, based on the priority of the packets. The SGW is also responsible for the mapping of the Quality of Service parameters between the aggregation network and the core network. We focus on an architecture with a single SGW, architectures with multiple SGWs and the handoff aspects between them will be reported upon in a later publication.

The remainder of this paper is structured as follows. In the following section different realistic scenarios for deploying the FAMOUS concept are identified. Next, the specific problems and technical solutions for the access network (Section 4) and the aggregation network (Section 5) are discussed. Finally, end conclusions are summed up.

\section{Scenarios: train, car}

Within the FAMOUS network architecture, we consider two scenarios: the train scenario and the car scenario, each with their specific characteristics. We further suppose that future broadband wireless technologies should be able to deliver DSL-like bandwidth to the users. This means that with an average of three active users per car, we need $10 \mathrm{Mb} / \mathrm{s}$ downlink bandwidth per vehicle. However, a train transports much higher number of users, (typically 1500 passengers a train), implying that the bandwidth requirements can mount to several $\mathrm{Gb} / \mathrm{s}$ per train. If we assume that only $20 \%$ of them want to make use of the broadband facilities, then we have 300 users per train or $1 \mathrm{~Gb} / \mathrm{s}$ downlink bandwidth per train. With an increasing user percentage, a need for several $\mathrm{Gb} / \mathrm{s}$ per train is not unrealistic. We further also have to consider the uplink traffic, which will for future multimedia applications, in particular for interactive applications (like video conferencing and gaming), be much more symmetric than current multimedia traffic.

Apart from the bandwidth demands, there are other differences between car and train scenario. In an urban environment, the different cars are much more geographically spread than the trains, which can only follow predefined trajectories on the rail tracks. Furthermore, train passengers are concentrated in a limited area along the rail track, while cars are more evenly distributed. Finally, in a train scenario, the users move all at the same velocity, while in car scenario each car has its own speed. This means that both the access network and the aggregation network have to deal with very different bandwidth demands for cars and for trains.

Given the different bandwidth requirements, the specific characteristics of each vehicle type and the limitations of the Mobile IP handoff protocol, we propose a different dedicated solution for fast handoff in 
the car scenario and the train scenario. They both try to solve the fundamental problem related to user mobility, namely handoff between base stations, but use a different technique taking into account the unique features of each environment.

\section{Access Network}

In case of frequent handoffs, Mobile IP suffers from several weaknesses like handoff latency, handoff packet loss and the load of control messages in the core network. Therefore, Mobile IP is proposed as a macromobility protocol to support the movements from one access network to another. Within one access network, a Mobile IP compatible micromobility handoff protocol is used. Such a micromobility protocol restricts the load of the control messages (needed to set up a new path after handoff) to that access network and aims to reduce handoff latency and packet loss. It handles local mobility locally, without informing the home agent or any correspondent node. Much research has been done in this area:

- Within the IETF, per-host forwarding schemes, like Cellular IP [13] and Hawaii [14], and tunnel-based protocols, like Mobile IPv4 Regional Registration [15] and Hierarchical Mobile IPv6 (HMIPv6) [16] were developed. These protocols assume a hierarchical access network and are therefore not able to handle extra links between access network routers in an optimal way.

- The BCMP micromobility protocol [17], developed within the IST BRAIN Project, is designed to operate irrespective of the access network topology. Authentication of the mobile host is executed by an anchor point, located in the access network. The handoff packet loss is reduced by the set up of a tunnel between the old and new access router. In addition, an optimal path between the anchor point and the new access router is obtained.

- Low Latency Handoff protocols, like Fast Mobile IPv6 (FMIPv6) [18], were developed by the IETF to reduce the amount of configuration time in a new subnet by using link layer triggers, in order to anticipate the movement of the mobile host. For example, a reduction in the signal strength of the downlink may indicate that handoff will be necessary soon. The protocol provides support for pre-configuration with link information of the new subnet, while the mobile host is still attached to the old subnet. Unfortunately, link layer triggers have not yet been standardized and are not always available in different access network technologies. 
The MIPv6 extensions, HMIPv6 and FMIPv6, are still in their initial experimental stage. The overall handoff latency and packet loss in MIPv6 will be dependent on the various delay factors such as movement detection, authentication and IP address configuration. The Mobile IP working group has not yet incorporated the case of a fast moving commuter demanding high bandwidth in their charter.

As the car and train scenario, described in Section 2, have different characteristics and requirements, each scenario asks for a different approach. The car scenario is characterized by very frequent handoffs, so fast handoff and route optimizations are required to guarantee a low handoff packet loss and limit the delay of data packets between the AGW and the access routers. The use of location information succeeds to further reduce the packet loss. In the train scenario, the amount of requested bandwidth is much higher and more concentrated. In order to support this scenario, we present a Radio-over-Fiber network in combination with moveable cells.

\subsection{CAR SCEnario}

As in the near future carpooling and public transport will be more and more encouraged by the local and public authorities, there will be an increasing demand for various Internet, multimedia and intelligent transportation applications in the car. For these kinds of applications, a high speed, high capacity wireless link is needed with a minimum handoff delay and minimal packet losses during handoff [19].

\subsubsection{Fast handoff with route optimizations}

Most research in the area of micromobility assumes that the links of the access network form a tree topology. However, for reasons of robustness against link failures and load balancing, a much more meshed topology is required. Some existing micromobility protocols, like Cellular IP and Mobile IPv4 Regional Registration do not take advantage of the mesh links to reduce the handoff latency and packet loss. By others, like Hawaii, the use of mesh links results in suboptimal routes and high end-to-end delays after several handoffs. In case of the BCMP protocol, no assumptions about the access network topology are made. However, when a mobile host moves far away from its anchor point and decides to change to another anchor point within the same access network, this inter-anchor handoff is a macromobility event.

A study of existing micromobility protocols resulted in a list of requirements we want to fulfill to obtain good local mobility support. To which extent these requirements are fulfilled is indicated by Table II. 
Table II. This table indicates how well the requirements for micromobility support are met by Cellular IP (CIP), Hawaii (HAW), MIPv4 Regional Registration (MRR) and the Brain Candidate Mobility Protocol (BCMP).

\begin{tabular}{|l|c|c|c|c|}
\hline & CIP & HAW & MRR & BCMP \\
\hline control load near access routers & no & yes & no & \pm \\
\hline low latency and low packet loss & \pm & yes & no & yes \\
\hline optimal paths (minimum hop count) & yes & no & yes & no \\
\hline handoff acknowledgments & no & yes & yes & yes \\
\hline transparent to mobile host & no & yes & \pm & no \\
\hline link failure robustness and support of QoS & no & no & no & no \\
\hline
\end{tabular}
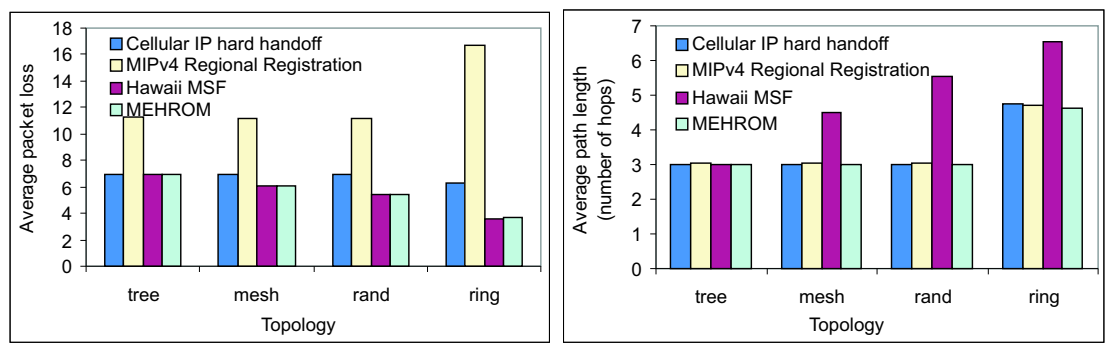

Figure 2. Results of MEHROM and comparison with other micromobility protocols for different access network topologies. The left figure compares the handoff packet loss, the right figure the path length in the access network.

We developed the micromobility handoff scheme MEHROM [20] [21], which stands for Micromobility support with Efficient Handoff and Route Optimization Mechanisms. This is a per-host forwarding scheme consisting of two phases. During the first phase, a fast handoff is made by sending a route update message towards the previous access router in a hop-by-hop way. The cross-over node does not forward the route update message further and is capable to detect whether the new path is optimal. If this phase results in an suboptimal path, a second phase, the route optimization phase, guarantees the set up of an optimal new path, by sending another route update message towards the AGW in a hop-by-hop way. The routers of the access network run OSPF (Open Shortest Path First) to obtain information about the next hop to a certain access router or the AGW.

MEHROM has a good performance for frequent handoffs in an IP domain, irrespective of the topology (pure tree, mesh, random, ring). Figure 2 shows that MEHROM has a lower handoff latency and packet loss than Cellular IP and Mobile IPv4 Regional Registration (left fig- 
ure). In contrast to Hawaii, MEHROM results in optimal paths between the AGW and the current access router (right figure).

\subsubsection{Location assisted handoff}

We are currently developing a dedicated intelligent routing protocol called APACHE (A Pro Active Handoff Enhancing protocol) [22] for fast and seamless handoff, taking into account the knowledge of the position and the trajectory of the vehicle. The latter can be accomplished for example by using GPS information. In APACHE location information is an integral part of the routing process in the access network, while current mobile networks only use it occasionally for location based services. Within the APACHE access network, the IP

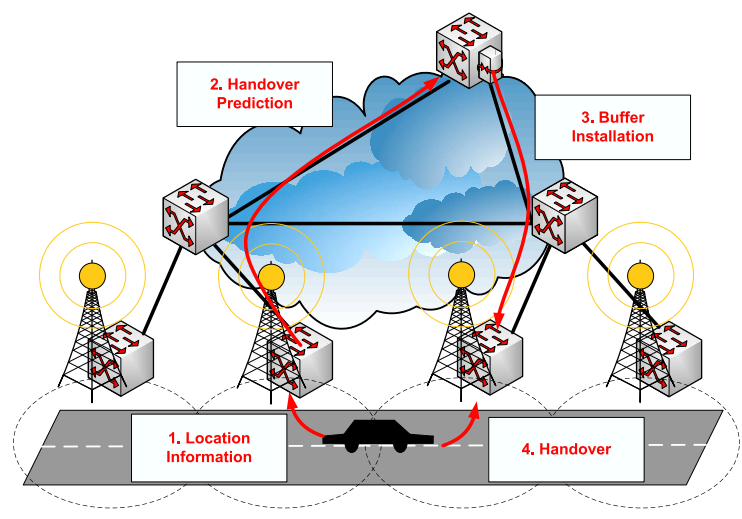

Figure 3. APACHE protocol

address of the mobile host does not change and mobility is handled at the ethernet level by dynamic ethernet tunnel management. Between heterogeneous access networks Mobile IP will be used to ensure the IP level connectivity of the vehicle.

In the APACHE protocol, the handoff instant is calculated and predicted at the current base station, using the location information from the vehicle (1). This information is relayed to the crossover switch, which is the switch or router common to both paths from the access network gateway to the old base station and to the new base station (2). At the calculated handoff instant, a buffer is installed at the next base station and routing is adapted, such that new arriving packets are directed towards that base station (3). After the actual physical and link layer handoff, packets are immediately available for the mobile host at the next base station. While current handoff protocols use packet forwarding from the old to the new base station and thereby increasing the handoff delay (or packet reordering), the APACHE protocol is pro- 
active in its routing and reduces the handoff delay to the time to synchronize, associate and authenticate the user at the base station. Figure 4 shows that APACHE is able to achieve a much higher TCP
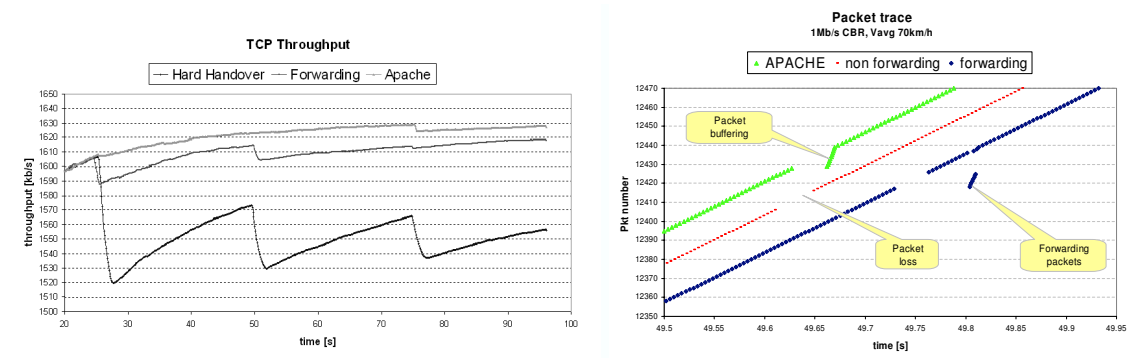

Figure 4. Results of APACHE TCP throughput and comparison with other handoff protocols

throughput than non forwarding (hard handoff) schemes, like Cellular IP, and forwarding schemes, like Hawaii. Additionally, APACHE does not introduce packet reordering like Hawaii, nor does it introduce packet loss like Cellular IP. It is therefore able to achieve much better performance than existing handoff protocols, making high quality multimedia services possible, even for fast moving mobile users.

\subsubsection{Quality of Service support}

The increasing use of wireless networks and the popularity of multimedia applications, leads to the need of Quality of Service support in a mobile IP-based environment. Users want to receive the same quality as in a fixed environment. QoS support in mobile and wireless IP networks is a very active research topic.

Current work within the IETF Next Steps In Signaling working group includes the analysis of existing QoS signaling protocols for an IP network and the listing of Mobile IP specific requirements of a QoS solution. The intention is to reuse, where appropriate, the protocol mechanisms of RSVP (Resource Reservation Protocol), while at the same time simplifying it and applying a more general signaling model. The use of RSVP in combination with mobility leads to several challenges. Mechanisms are needed to minimize the resource reservation delay and the handoff packet loss. Old reservations must be released and resources along the common part of the old and new path must not be allocated twice.

Developed within the IETF Seamoby working group, the CARD (Candidate Access Router Discovery) protocol enables the mobile hosts to discover the identities of candidate access routers and their capabilities prior to the initiation of the IP-layer handoff. The CTP (Con- 
text Transfer Protocol) enables authorized context transfers to avoid re-initiation of signaling to and from the mobile host after handoff.

The MEHROM and APACHE protocols will be enhanced in order to be able to guarantee the necessary Quality of Service in the vehicle's access network:

- Currently, Q-MEHROM is developed, which is the close coupling of MEHROM and a resource reservation mechanism. In case of handoff, the updating of routing information and the allocation of resources are performed at the same time. Invalid routing information and reservations along the old path are explicitly deleted, and resource reservations along the part of the old path that overlaps with the new path are reused. Hereby, QoS extensions to OSPF are used to obtain information about available resources in the access network.

- The APACHE protocol requires scheduling techniques to ensure the quality of service requirements for each of the mobile host's applications at various vehicular speeds. The APACHE protocol will also be extended towards complex environments with high user density, like cities, where resource reservation is a key issue in order to guarantee the requested QoS.

\subsection{TRAIN SCENARIO}

As described in Section 3, the desired bandwidth is much higher in a train scenario. To meet this requirement, we propose an architecture consisting of a Radio-over-Fiber (RoF) network in combination with moveable cells. These two concepts take advantage of some specific aspects of a train scenario, such as equal speed of all users and concentration of the train passengers along the railway.

Along the rail tracks, Remote Antenna Units (RAUs) are put at a fixed distance from each other, and in that way they form a cellular network. On the roof of the train, a number of antennas will be evenly distributed and a wireless connection will be set up between the RAUs and the antennas on the train. The latter will feed an indoor LAN (wired or wireless): this two-step approach avoids high penetration losses between the RAU and end users.

To be able to offer a broadband connection, small cells (even smaller than in the car scenario, e.g. 50 to $100 \mathrm{~m}$ in diameter) and consequently a large number of RAUs are required. Furthermore, also a high radio frequency out of the millimeter wave frequency range (e.g. 17, 28 or 60 $\mathrm{GHz}$ ) may be essential to offer the desired bandwidth. 
4.2.1. Radio-over-Fiber network

In such a situation - combination of small cells and high radio frequencies - a Radio-over-Fiber (RoF) system is very attractive [23]. The goal of a RoF network is to transfer complicated microwave and millimeter wave signal processing functions from the RAUs to a centralized control station. In this control station, the data will be modulated on the right radio frequency. Then, the radio signals will be converted to the optical domain and finally, they will be transmitted by an optical fiber to the RAUs. The latter only have to recover the radio signal, which can be transmitted immediately without any further processing. In this way, the expensive signal processing equipment at the control station can then be shared among a large number of RAUs and all intelligence will be situated at the control station. Each RAU becomes then a passive device and is thus significantly simplified, which is a critical issue in high-frequency systems.

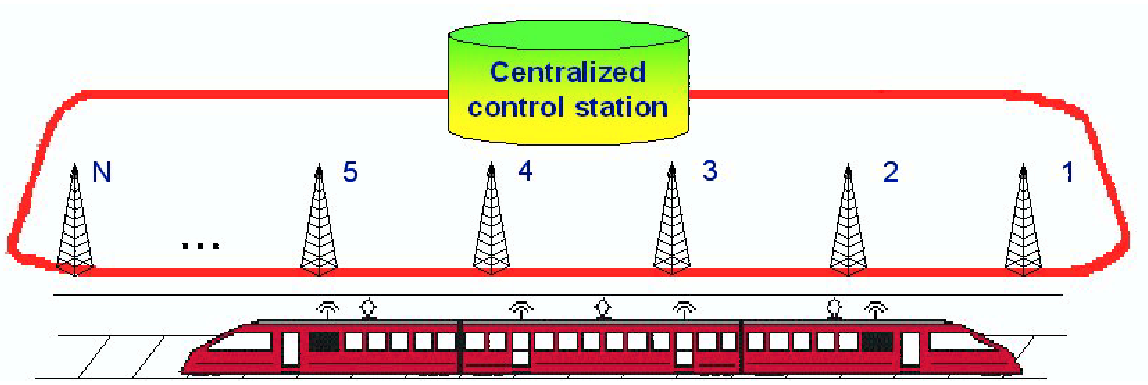

Figure 5. Illustration of a Radio-over-Fiber network: RAUs along the railway, together with centralized control station.

We also draw attention to the fact that a train is connected to more than one RAU at each moment. All the RAUs in the reach of the train could receive another radio signal, and thanks to the use of Wavelength Division Multiplexing (WDM) each radio signal can be provided by a dedicated wavelength. Furthermore, all train users are concentrated on a limited area along the railway, while there are typically no users in front of or behind the train. By consequence, the same wavelengths can then be reused for the RAUs in front of and behind the train. In this case, it is advisable to feed the RAUs within range of the same control station by one optical fiber. Considering the resilience aspects of the network, a ring is a very promising topology (see Figure 5).

\subsubsection{Moveable cells to reduce the handoff times}

There will be a continuous need for handoffs when moving along the RAUs. However, instead of having the train moving along a fixed repeated cell pattern, one might also consider reconfiguring the optical 


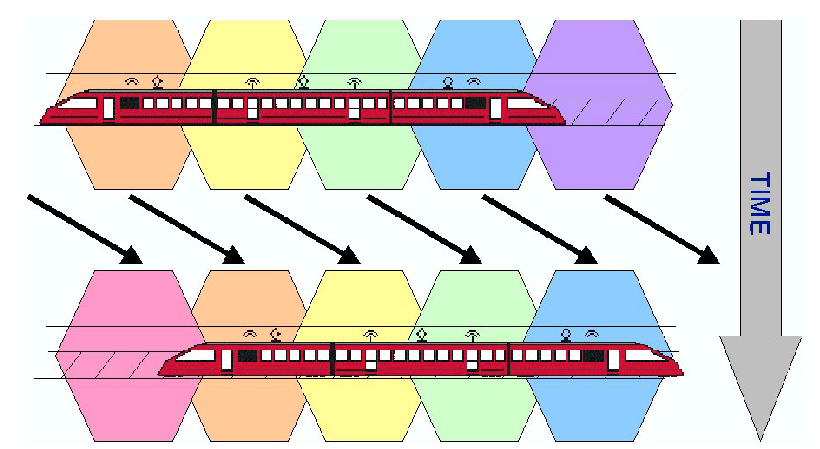

Figure 6. Illustration of the moveable cell concept.

feeder network feeding the RAUs along the rail track in order to have a cell pattern that moves together with the train (see Figure 6). This way the latter can communicate on the same frequency during the whole connection and also avoid (most of) the cumbersome handoffs. It is clear that synchronizing the speed of the cells with that of the train is of utmost importance and that the required reconfigurations should be kept as short as possible. This concept of moveable cells is extremely attractive in a train scenario, because all users move at the same speed.

To understand the advantage of the moveable cell concept, consider the situation where we do not use this concept. In this case, the data flow has to be put on another frequency each time the train comes in the range of another RAU. This means, in the control station, the data flow has to be switched to another base station, and thus a switchover is inevitable. Furthermore, without moveable cells, the antennas on the train also have to change their frequency. This means it will be necessary to synchronize the switchover in the control station with the one on the train, which can be very time-consuming. That is the reason why classical handoff times often have an order of magnitude of 1 second. The moveable cell concept also takes advantage of the RoF technology, because the switchover can be completely performed in a central way. Classically, without RoF, the base stations (situated along the rail track) will receive the information in a digital form. Using moveable cells without RoF means we have to send the digital information to another base station, and the RF $\mathrm{Rx} / \mathrm{Tx}$ module in the base station has to switch to another frequency. This will again require synchronization between both switchovers.

\subsubsection{Optical Switching Architecture}

The reconfiguration to implement the moveable cells will take place entirely in the optical domain. A possible implementation of this moveable cell concept is illustrated in Figure 7 and already presented in [24]. The 


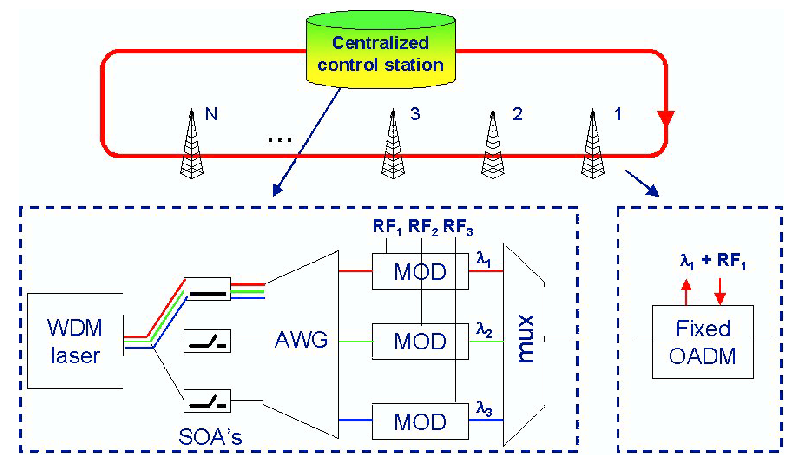

Figure 7. Possible optical switching architecture to implement the moveable cell concept.

intention is to accomplish the moveable cells by wavelength switching in the central control station. If in each RAU, there is installed a fixed Optical Add Drop Multiplexer (OADM), then in each RAU, a fixed wavelength is terminated. The idea is then to put the desired frequency for a certain RAU on the right wavelength. This can be done through the use of some optical switches in combination with a WDM laser and an Arrayed Waveguide Grating (AWG).

The WDM laser will generate a beam of light containing the desired wavelengths. The entire beam is sent to a set of optical switches (e.g. Semiconductor Optical Amplifiers (SOAs) used as optical gates) and as explained further in this section, only one of them will let pass the beam. With the help of an AWG, the different wavelengths can be spread out in a required order and each output port of the AWG is then connected with a different RF modulator (each generating another radio frequency). Finally, the modulated wavelengths leaving the RF modulators are multiplexed.

The order of wavelengths leaving the AWG will be dependent on the input port where we offer the beam of light. Moreover, an AWG has a cyclic nature: if the beam of light enters input port 1 , and the corresponding output sequence is: $\lambda_{1}, \lambda_{2}, \ldots \lambda_{m}$, then if the beam enters input port 2 , the output sequence will be: $\lambda_{m}, \lambda_{1}, \ldots \lambda_{m-1}$. The wavelength leaving a certain output port of the AWG will always be modulated by the same RF signal. To modulate a certain wavelength with the right RF signal, this wavelength has to leave the right output port of the AWG. That is why we equip each input port of the AWG with an optical switch, then we can obtain the desired wavelength order at the output ports of the AWG by closing the right switch so that the beam of light enters the right input port. On the example of Figure 7, $\mathrm{m}=3$ and the first SOA is in use, which means the beam of light 
enters input port 1 , and in this case the output order is $\lambda_{1}, \lambda_{2}, \lambda_{3}$. By consequence $\lambda_{1}$ is modulated with $R F_{1}$ and so on. The RAU which is equipped with the OADM which drops $\lambda_{1}$ will transmit its information on $R F_{1}$.

\subsubsection{Benefits of the moveable cell concept}

Classical handoff times of e.g. $100 \mathrm{~ms}, 500 \mathrm{~ms}$ or $1 \mathrm{~s}$ are not an exception (cf. Mobile IPv6 protocol with handoff times in the order of seconds), but rather the general rule. To see why a handoff time of e.g. 1s is not sufficient, consider the next simple example: a cell range of $100 \mathrm{~m}$ combined with a train speed of $160 \mathrm{~km} / \mathrm{h}$ means a handoff every $2.25 \mathrm{~s}$. In combination with a handoff time of $1 \mathrm{~s}$, we achieve a loss of $44 \%$, which is unacceptable.

Table III. Some optical switches with their switching times.

\begin{tabular}{|c|c|c|}
\hline no. & switch & switching time \\
\hline 1 & SOA, electrooptic & $5 \mathrm{~ns}$ \\
\hline 2 & acoustooptic & $3 \mu \mathrm{s}$ \\
\hline 3 & thermooptic, electromechanical, liquid crystal & $4 \mathrm{~ms}$ \\
\hline 4 & MEMS, bubble & $10 \mathrm{~ms}$ \\
\hline
\end{tabular}

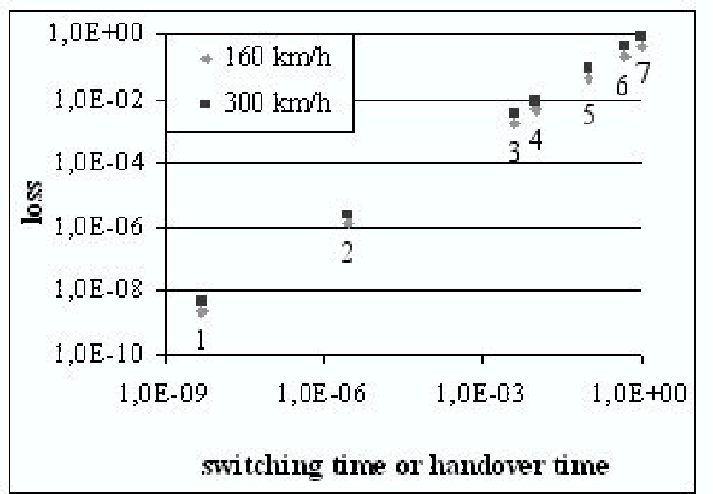

Figure 8. Loss in function of the different switching or handoff times

In our proposed architecture, we can assume that the switching time of the optical switches is the most time consuming factor. If we suppose the total handoff time is equal to this switching time, then we have a first approximation of the handoff time in case of the moveable cell concept. Table III gives an overview of some important optical switches. So, we see the handoff times can be reduced considerably. 
Figure 8 shows the losses in case of cell range of $100 \mathrm{~m}$, a train speed of $160 \mathrm{~km} / \mathrm{h}$ and $300 \mathrm{~km} / \mathrm{h}$, and this in function of the different optical switching times or handoff times. No. 1-4 corresponds to the switches of Table III. No. 5, 6 and 7 respectively correspond to $100 \mathrm{~ms}, 500 \mathrm{~ms}$ and $1 \mathrm{~s}$.

\section{Aggregation Network}

While fast moving vehicles are travelling from access network to access network, the aggregation network is providing the connectivity between these access networks and the SP's network. The Aggregation Network Providers (ANPs) would like an efficient way for designing and managing this aggregation network. In this section we will propose a design method for an aggregation network that is able to provide multimedia services to fast moving users in an efficient way.

The fast moving aspect implies that the network is under rapidly changing traffic conditions. In the classic network design broadband networks are not optimally designed to cope with fast moving users. Such a design (even with addition of an admission control system to limit the impact of unexceptional circumstances) won't be sufficient to maintain the QoS guarantees of the passenger data traffic at all times without overdimensioning the network. The data traffic must be delivered with the required QoS guarantees along the entire trajectory of the users and overdimensioning is not what the ANPs desire.

More efficient would be an aggregation network that changes dynamically according to the current positions of the moving vehicles. This requires a specific management system that automatically configures the network. This management system will consist of two major parts (represented on Figure 9): an online part and an off-line part. On one hand the online management system deals with the service realization and on the other hand the off-line management system deals with the network capacity dimensioning and optimal route determination. Mainly due to economical reasons ANPs tend towards cost efficient networks consisting of standard QoS-aware Ethernet switches (IEEE 802.1s, IEEE 802.1q\&p compliant) instead of IP/MPLS-based architectures. To achieve the high service levels that are required for FAMOUS, some issues remain to be solved. 


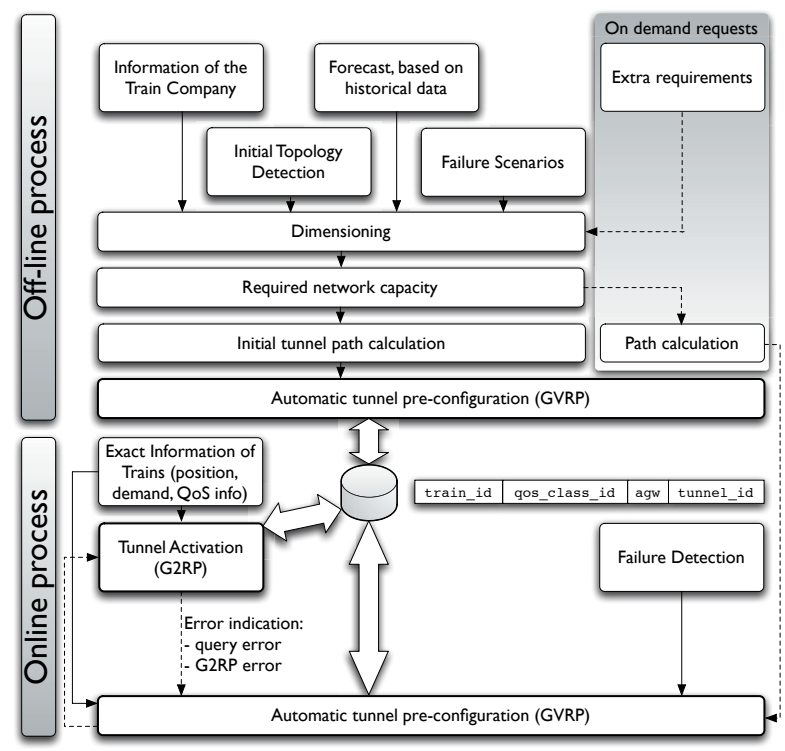

Figure 9. Management system

\subsection{Online Process: Service Realization}

\subsubsection{Service Platform Architecture}

The aggregation network is responsible for the transport of the passenger traffic by means of high bandwidth tunnels between the access network and the SP domain. Therefore, the passenger traffic is gathered in aggregated traffic flows per vehicle and per QoS class, whereas in the access network traffic demands of the users were handled individually. At the AGWs the aggregated traffic flows are mapped into dynamic tunnels. In our architecture, the physical realization of these tunnels is established by point-to-point Ethernet VLANs (Virtual LANs). The VLAN technology allows the management system to make optimal use of the MSTP (Multiple Spanning Tree Protocol, IEEE 802.1s). In this way network resources are optimally utilized and Traffic Engineering (TE) can be applied [25]. The establishment of the physical tunnels is not sufficient to guarantee end-to-end QoS for the transported data traffic. Therefore, reservation states associated with these tunnels will be maintained and an admission control mechanism will limit the amount of allowed data traffic. To remove the burden of manually installing VLANs from the network administrator's hands and to impose the admission control's regulations under rapidly changing traffic conditions, a dedicated management system is required. This management system is also responsible for generating triggers based 
on exact information such as train positions, increase in amount of passengers, etc. and for reconfigurating tunnels in case of network element failures or when the set of pre-configured tunnels are insufficient to carry the instantaneous demand. Two protocols have been developed which allow automatic VLAN tunnel setup and distribution of reservation states. These protocols allow easy plug-in of the other management components.

\subsubsection{Design of Tunnel Set-up Protocol for Switched Ethernet}

Existing tunnel mechanisms for IP/MPLS networks (such as LDP, RSVP and their extensions) are not easily portable to Switched Ethernet networks. To avoid designing brand new protocols, we used standardized GARP (Generic Attribute Registration Protocol) protocols. GARP, described in IEEE 802.1d, provides a generic attribute dissemination capability for bridged LANs. For the establishment of the VLAN tunnels we specifically developed a "Scoped Refresh" extension of the standardized GVRP (Generic VLAN Registration Protocol) protocol. This extension resolves the relevant problem of depletion of the limited sized VLAN databases. For distributing the reservation parameters associated with these tunnels, a new GARP application is developed with closed loop design, mechanism for updating reservation parameters and hop-by-hop admission control. The new protocol is referred to as G2RP (Generic Reservation Parameters Registration Protocol) and is used to activate the required VLAN tunnels according to the position and movement of the connected vehicles. The SGW will maintain a tunnel mapping database to direct the traffic destined for the vehicles into the corresponding VLAN tunnel. This database will get updated with current locations of the vehicles. By separating the distribution of reservation parameters and VLAN IDs, the required VLAN tunnels can be pre-configured separately while G2RP can be used to activate the required tunnels at their due time and de-activate the tunnels when no longer required. On Figure 10 the performance of the VLAN
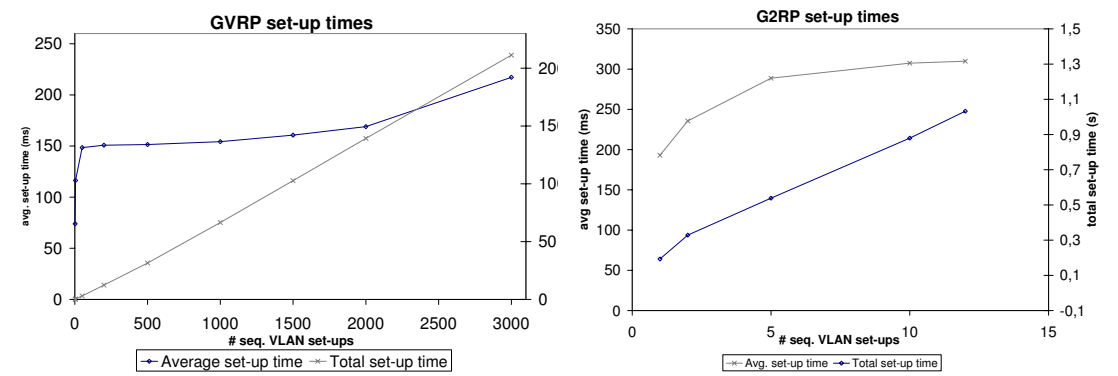

Figure 10. Pre-configuration times and activation times 
pre-configuration and the tunnel activation are presented. For further details about the protocol design, implementation aspects or evaluation results the reader is kindly referred to [26].

\subsubsection{Resilience issues}

Aggregation networks will cover large areas such as entire railroad tracks or national road infrastructures and network links will carry a lot of data traffic (typically $1 \mathrm{~Gb} / \mathrm{s}$ or $10 \mathrm{~Gb} / \mathrm{s}$ Ethernet line systems). This means that long term outage of a link effects a large range of users. Therefore, a fast and efficient resilience mechanism is required. The performance of Spanning Tree Protocol extensions such as the Rapid Spanning Tree Protocol (IEEE 802.1w) and MSTP (IEEE 802.1s) and their interworking with GVRP/G2RP will be examined. These resilience mechanisms will cause tunnel reconfigurations and the deployment of a specifically designed resilience strategy will be investigated.

\subsection{Off-line Process: Cost efficient Capacity Assignment and Path Determination of the Dynamic Tunnels}

The main problem, tackled by the off-line process, can be described as follows: how to calculate and set up dynamic tunnels between the gateways in the aggregation network to meet the traffic demand requests while achieving low congestion and optimizing the utilization of the network resources. The complexity of this problem is illustrated on a simple network example with three AGWs and a single SGW. Assume that the aggregation network needs to support a single train with 2 $\mathrm{Gb} / \mathrm{s}$ load or two crossing trains with $1 \mathrm{~Gb} / \mathrm{s}$ load each. A first option would be to design the network intuitively: namely in a way that every AGW is connected with the SGW along its shortest path (bottom left on Figure 11). In this way the lowest average hop count is achieved but with an expensive topology cost, certainly if you assume that in many cases fibers are already present along the railroad. In case of dominant topology cost, a second design with the cheapest topology cost but high average hop count is proposed (bottom right on Figure 11). However if the node cost becomes increasingly important (e.g. in the case that all fibers are already in place), both previously presented solutions had a interface cost of $12 \mathrm{~Gb} / \mathrm{s}$ but the solution with the cheapest node cost has an interface cost of only $8 \mathrm{~Gb} / \mathrm{s}$ (top on Figure 11). In other words, depending on the business case and the current network status the design of aggregation networks of realistic size requires a more methodical approach. Not depending on the specific deployed network technology, a generic mathematical model is designed that determines 


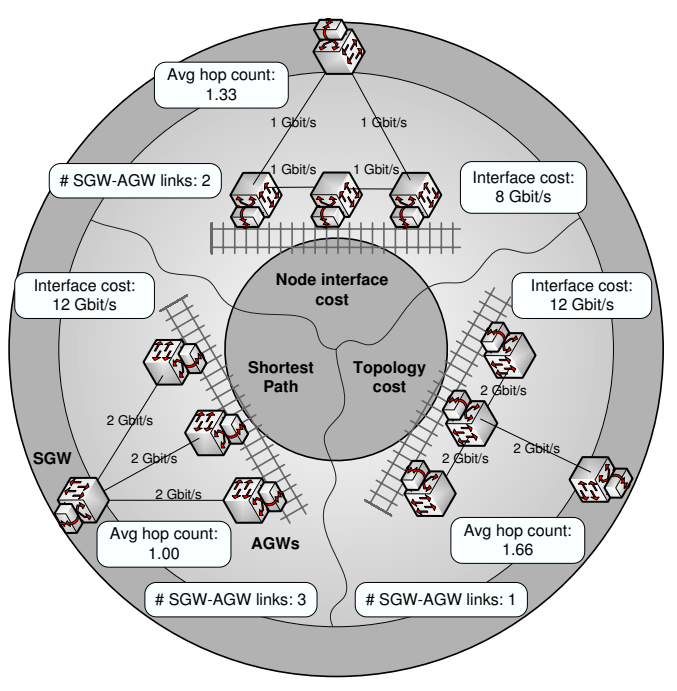

Figure 11. Illustration of the network design complexity for a scenario with a single train with $2 \mathrm{~Gb} / \mathrm{s}$ load or two crossing trains with $1 \mathrm{~Gb} / \mathrm{s}$ load each.

the network equipment that needs to be installed and where to place the fibers between the network nodes, how to set up and adjust the AGW-SGW tunnels and how to optimally route the traffic flows for every anticipated situation.

In [27] the routing and dimensioning issues of time-scheduled connections in QoS networks are addressed but for FAMOUS a model is specifically designed to take the needs of fast moving users into account (expressed by strongly time-dependent inputs and constraints). This leads to a complex problem formulation that can be solved by using ILP solving techniques. This is elaborated in [12]. Without loss of generality the evaluations in [12] focussed on train scenarios. Traffic demands can be derived from train schedules, train delays, predictions about increase of the amount of commuters and the anticipated failure scenarios. But the traffic demand derivation is important for the network design outcome. Static traffic demands that neglect all timerelated aspects about the moving aspect of the vehicles, result in an overdimensioning of the network. Exact traffic demands as predicted by the train schedules can easily be derived from the input information but the dimensioning results in a cost optimized network that is very sensitive to train delays. If crossing trains are running $90 \mathrm{~km} / \mathrm{h}$ on the average and if AGWs are positioned along a linear railroad every $10 \mathrm{~km}$, the train delays that can be covered, vary from $0 \mathrm{sec}$ (worst case) up to 3 min20sec. Bigger train schedule changes or larger train delays 
cause service interruptions along the passenger's trajectory. Therefore an alternative demand model was developed that leads to train delay insensitive (TDI) network dimensioning. In [12] it is detailled that TDI dimensioned networks are significantly cheaper solutions compared to statically overdimensioned networks and the extra cost with respect to exact dimensioned networks remains limited.

Because of the increased complexity of the TDI dimensioning, the dimensioning problem becomes hard to solve for networks of realistic size. Therefore, heuristic approaches will be developed. Service realization of optimized traffic engineering strategies and resilience strategies are topics for future work. In our test lab, a Linux test bed will be built with basic management system to implement the concept and evaluate the QoS level experienced by moving users. This part of the FAMOUS project intends to contribute in the general ongoing research of extending the Ethernet standard towards a true transport technology for aggregation networks.

\section{Conclusion}

In this paper we have presented an architectural framework for the provision of broadband applications to fast moving users in the car or in the train. We have further shown that within the proposed architecture from a technological point of view there may exist several solutions to enable multimedia services to fast moving mobile users. However, a lot of issues still remain unresolved and require further parallel studies and research. Prior to broadband services on fast moving terminals become a success story, a good business model is needed. Such a model has to find answers to which players (network operators, service providers, train operators, etc.) are involved, what kind of partnerships needs to be established between the players in order to offer seamless services over different operator owned networks, which is the best pricing policy, how much the mobile user wants to pay for which mobile services and applications, etc. Another issue which certainly needs further attention is how to protect the privacy of the mobile user and how to ensure secure connectivity.

\section{Acknowledgements}

This research is funded by a Ph.D. grant for L. Peters of the Fund for Scientific Research - Flanders (F.W.O.-V., Belgium) and by Ph.D. grants for T. Van Leeuwen and F. Van Quickenborne of the Institute 
for the Promotion of Innovation through Science and Technology in Flanders (IWT-Vlaanderen). D. Colle thanks the IWT for its financial support for his postdoctoral grants. Part of this research is also funded by the Belgian Science Policy Office (BelSPO, Belgium) through the IAP (phase V) Contract No. IAPV/11, by Ghent University through the project BOF-RODEO and by the Institute for the promotion of Innovation by Science and Technology in Flanders (IWT, Flanders) through the GBOU Contract 20152 "End-to-End QoS in an IP Based Mobile Network".

\section{References}

1. International Telecommunication Union. Information Technology Statistics http://www.itu.int/ITU-D/ict/statistics/at_glance/Internet03.pdf, 2003.

2. Internet World Stats Internet Usage Statistics - The Big Picture World Internet Users and Population Stats. http://www.internetworldstats.com/stats.htm

3. Point Topic Ltd. World Broadband Statistics: Q4 2003. http://www.pointtopic.com/contentDownload/freeforyou/world\%20broadband\%20statistics\%20q4 \%202003.pdf, website, 23 March, 2004.

4. GSM World from the GSM Association. Growth of the global digital mobile market. http://www.gsmworld.com/news/statistics/pdf/gsma_stats_q2_04.pdf, website, 2004.

5. Connexion by BoeingSM. http://www.boeing.com/connexion/flash.html, website.

6. GNER, Icomera announce commercial agreement to deliver real-time wireless internet on trains. http://www.icomera.com/news/gner_agreement.asp, website, Gothenburg, Sweden, 6 April, 2004.

7. Irvine, J., D. Robertson and J. Dunlop. The MOSTRAIN (mobile services for high speed trains) system demonstrator. Personal, Indoor and Mobile Radio Communications, 1998. The Ninth IEEE International Symposium on, vol. 2, 8-11, pp. 1004-1008, September 1998.

8. Ott, J. and D. Kutscher. Drive-thru Internet: IEEE 802.11b for Automobile Users. IEEE Infocom 2004 Conference, Honk Kong, March 2004.

9. Fleishman, G. Destination wi-fi, by rail, bus or boat. The New York Times, New York, July 2004.

10. An Intelligent Wi-Fi Bus Equipped with Appear Networks Technology in Paris. http://www.appearnetworks.com/press_room/press_releases/pr_ratp_bus _june2004.html, website, Stockholm, Sweden, 11 June 2004.

11. Johnson, D., C. Perkins and J. Arkko. Mobility Support in IPv6. IETF RFC 3775, June 2004.

12. Van Quickenborne, F., F. De Greve, F. De Turck, P. Demeester et al. Cost Effective Design of Aggregation Networks for Fast Moving Vehicles. The 11th Symposium on Communications and Vehicular Technology in the Benelux: SCVT 2004, Gent, Belgium, November 2004.

13. Valkó, A. Cellular IP: A New Approach to Internet Host Mobility. ACM Computer Communication Review, January 1999. 
14. Ramjee, R., T. La Porta, L. Salgarelli, S. Thuel and K. Varadhan. IP-Based Access Network Infrastructure for Next-Generation Wireless Data Networks. IEEE Personal Communications, pp. 34-41, August 2000.

15. Gustafsson, E., A. Jonsson and C. Perkins. Mobile IPv4 Regional Registration. draft-ietf-mobileip-reg-tunnel-0\%.txt, October 2002 (work in progress).

16. Soliman, H., C. Catelluccia, K. El Malki and L. Bellier. Hierarchical Mobile IPv6 mobility management (HMIPv6). draft-ietf-mipshop-hmipv6-02.txt, June 2004 (work in progress).

17. Boukis, C., N. Georganopoulos and H. Aghvami. A hardware implementation of BCMP mobility protocol for IPv6 networks. GLOBECOM 2003 - IEEE Global Telecommunications Conference, vol. 22, no. 1, pp. 3083-3087, December 2003.

18. Koodli, R., Ed. Fast Handovers for Mobile IPv6. draft-ietf-mipshop-fast-mipv602.txt, July 2004 (work in progress).

19. Van Leeuwen, T., I. Moerman, B. Dhoedt and P. Demeester. Broadband Wireless Communication in Vehicles. $42^{\text {nd }}$ European Telecommunications Congress (FITCE 2003), pp. 77-82, 4-6 September, Berlin, Germany.

20. Peters, L., I. Moerman, B. Dhoedt and P. Demeester. Micro-Mobility support for random access network topologies. IEEE Wireless Communication and Networking Conference (WCNC 2004), 21-25 March, Georgia, USA, ISBN 07803-8344-3.

21. Peters, L., I. Moerman, B. Dhoedt and P. Demeester. MEHROM: Micromobility support with efficient handoff and route optimization mechanisms. $16^{\text {th }}$ ITC Specialist Seminar on Performance Evaluation of Wireless and Mobile Systems (ITCSS16 2004), pp. 269-278, 31 August-2 September, Antwerp, Belgium.

22. Van Leeuwen, T., I. Moerman, B. Dhoedt and P. Demeester. A Pro-active Handoff Enhancing protocol. IST Mobile and Wireless Summit 2004, pp. 844848, 27-29 June, Lyon, France.

23. Al-Raweshidy $\mathrm{H}$. et al, Radio over Fiber Technologies for Mobile Comm. Networks, Artech House Inc., Norwood, USA, 2002.

24. Lannoo B., D. Colle, M. Pickavet, P. Demeester, Optical Switching Architecture to Implement Moveable Cells in a Multimedia Train Environment. Proceedings of the 30th European Conference on Optical Communication (ECOC 2004), Stockholm, Sweden, September 2004.

25. Srikant, S., G. Kartik et al. Viking: a Multi-Spanning Tree Ethernet Architecture for Metropolitan Area and Cluster Networks. The 23rd Conference of the IEEE Communications Society: Infocom 2004, Hong Kong, China, March 2004.

26. De Greve, F., F. Van Quickenborne, F. De Turck, P. Demeester et al. Evaluation of a Tunnel Set-up Mechanism in QoS-aware Ethernet Access Networks. Proceedings of the 13th IEEE Workshop on Local and Metropolitan Area Networks: LANMAN 2004, San Francisco, USA, April 2004.

27. De Turck, F., P. Demeester and H. Alaiwan. Efficient bandwidth scheduling over switched QoS networks. The 8th Int. Telecommunication Network Planning Symposium, pp. 249-254, Sorrento, Italy, 18-23 October, 1998. 